\title{
Finding Double-Barred Galaxies with HST
}

\author{
T. Lisker ${ }^{1}$, V. P. Debattista ${ }^{2} \dagger$, I. Ferreras ${ }^{3}$, and P. Erwin ${ }^{4}$ \\ ${ }^{1}$ Astronomical Institute, Dept. of Physics and Astronomy, University of Basel, Switzerland \\ ${ }^{2}$ Astronomy Department, University of Washington, Seattle, USA \\ ${ }^{3}$ Department of Physics and Astronomy, University College London, UK \\ ${ }^{4}$ Max-Planck-Institut für Extraterrestrische Physik, Garching, Germany
}

\begin{abstract}
We show that the detection of double-barred (S2B) galaxies beyond the nearby universe is possible out to redshifts $0.1 \lesssim z \lesssim 0.5$ with the resolution of the HST Advanced Camera for Surveys. We present the most distant S2B currently known, at $z=0.148$.
\end{abstract}

Keywords. Galaxies: structure, galaxies: distances and redshifts, galaxies: evolution

Double-barred (S2B) galaxies could be a mechanism for feeding supermassive black holes by funneling gas to the center (Shlosman et al. 1990). Until recently, S2Bs had only been identified in the nearby $(z \leqslant 0.04)$ universe, which led us to perform a feasibility study for detecting and analysing them at intermediate redshifts (Lisker et al. 2006) with the HST Advanced Camera for Surveys (ACS) data of the Great Observatories Origins Deep Survey (Giavalisco et al. 2004). We identified the two most distant S2Bs known so far, at redshifts $z=0.103$ and $z=0.148$ (Figs. $1 \& 2$ ), corresponding to a look-back time of 1.3 and 1.9 Gyr, respectively. Based on local S2B sizes, deep HST/ACS and similar surveys have the potential to push the limit for $\mathrm{S} 2 \mathrm{~B}$ detection out to a look-back time of 5 Gyr. An S2B sample distributed over a large redshift range therefore seems possible in the near future, which would serve as important constraint on S2B formation models.

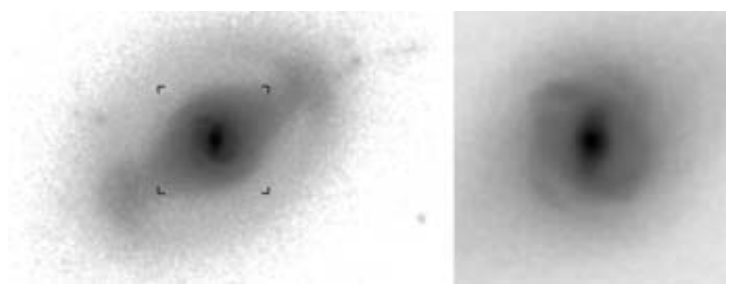

Figure 1. HST-GOODS J033230.93-273923.7 — a double-barred galaxy at $z=0.148$.

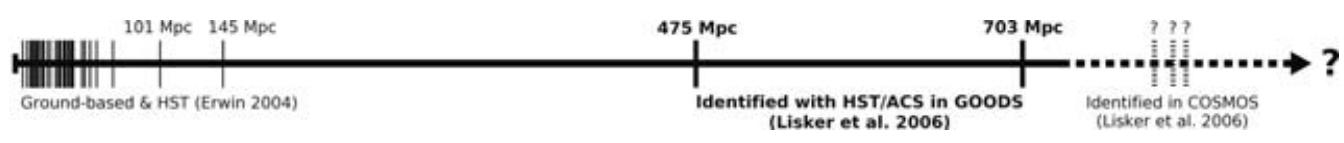

Figure 2. Distance to known double-barred galaxies.

\section{References}

Erwin, P. 2004, A\&A 415, 941.

Giavalisco, M., Ferguson, H.C., Koekemoer, A.M., et al. 2004, ApJ 600, L93.

Lisker, T., Debattista, V.P., Ferreras, I. \& Erwin, P. 2006, MNRAS 370, 477.

Shlosman, I., Begelman, M.C. \& Frank, J. 1990, Nature 345, 679.

\section{$\dagger$ Brooks Prize Fellow}

